\title{
Força muscular e densidade mineral óssea em idosos eutróficos e desnutridos
}

\author{
Muscle strength and bone mineral density in \\ well-nourished and malnourished elderly
}

Karla Helena Coelho VILAÇA ${ }^{1,2}$

Eduardo FERRIOLLI²

Nereida Kilza da Costa LIMA²

Francisco José Albuquerque de PAULA²

Julio Sérgio MARCHINI²

Julio Cesar MORIGUTI²

RE S U M O

\section{Objetivo}

Este estudo teve como objetivo avaliar a associação do estado nutricional com a força muscular de preensão manual e a densidade mineral óssea em idosos do sexo masculino.

\section{Métodos}

Participaram do estudo 41 idosos do sexo masculino, sendo 20 eutróficos (peso: $M=69,6, D P=8,4$; índice de massa corporal: $M=25,7, D P=2,2$ ) e 21 desnutridos (peso: $M=50,9, D P=6,1$; índice de massa corporal: $M=18,7$, $D P=1,8)$, classificados subjetivamente segundo a Mini Avaliação Nutricional. A avaliação antropométrica incluiu peso corporal, altura, circunferência do braço e da panturrilha. A composição corporal foi avaliada pelo método de absorciometria por dupla emissão de Raios X, e a ocorrência de osteoporose foi definida de acordo com os critérios estabelecidos pela Organização Mundial da Saúde. A força muscular foi avaliada pelo dinamômetro de mão.

\section{Resultados}

O grupo desnutrido apresentou valores expressivamente menores dos parâmetros antropométricos e de composição corporal em relação ao grupo eutrófico $(p<0,005)$. Além disso, a ocorrência de osteoporose foi significativamente maior $(p<0,01)$ no grupo desnutrido em todas as regiões estudadas: colo do fêmur, quadril total e coluna. A força muscular de preensão manual dos idosos desnutridos foi significativamente menor que a dos idosos eutróficos ( $M=23,1, D P=6,8$ e $M=30,3, D P=8,4$, respectivamente; $p<0,005)$.

\footnotetext{
${ }^{1}$ Universidade Católica de Brasília, Programa de Pós-Graduação Stricto Sensu em Gerontologia. Brasilia, DF, Brasil.

2 Universidade de São Paulo, Faculdade de Medicina de Ribeirão Preto, Departamento de Clínica Médica. Av. Bandeirantes, 3900, Monte Alegre, 14049-900, Ribeirão Preto, SP, Brasil. Correspondência para/Correspondence to: K.H.C. VILAÇA. E-mail: <karlav@ucb.br>.
} 
846 | K.H.C. VILAÇA et al.

\section{Conclusão}

A desnutrição está associada à menor força muscular e à diminuição da densidade mineral óssea em idosos do sexo masculino.

Termos de indexação: Composição corporal. Desnutrição. Envelhecimento. Força muscular. Idoso. Peso corporal.

\section{A B S T R A C T}

\section{Objective}

The present study investigated the possible association of nutritional status with handgrip strength and bone mineral density in elderly men.

\section{Methods}

The study included 41 elderly males of which 20 were well-nourished (weight: $M=69.6, S D=8.4 \mathrm{~kg} ; B M I: M=25.7$, $S D=2.2$ ) and 21 were malnourished (weight: $M=50.9, S D=6.1 \mathrm{~kg} ; B M I: M=18.7, S D=1.8$ ). They were subjectivity classified by the Mini Nutritional Assessment. Anthropometric measurements included weight, height and arm and calf circumferences. Body composition was determined by dual energy $x$-ray absorptiometry and presence of osteoporosis was determined according to the World Health Organization's criteria. Muscle strength was assessed by a handheld dynamometer.

\section{Results}

The malnourished group presented significantly lower anthropometric and body composition measurements than the well-nourished group $(p<0.005)$. Furthermore, the occurrence of osteoporosis was significantly higher $(p<0.01)$ among malnourished individuals in all studied body areas: femoral neck, total hip and spine. The handgrip strength of malnourished individuals was significantly lower than that of well-nourished individuals $(M=23.1, S D=6.8$ and $M=30.3, S D=8.4$, respectively; $p<0.005)$.

\section{Conclusion}

Malnutrition is associated with lower muscle strength and low bone mineral density in elderly men.

Indexing terms: Body composition. Malnutrition. Aging. Muscle strength. Aged. Body weight.

\section{N T R O D U Ç Ã O}

A desnutrição é um problema grave para o idoso, podendo ser decorrente de fatores socioeconômicos, culturais, fisiológicos, patológicos e cognitivos, capazes de levar ao aparecimento de diversas doenças e de associar-se à precária condição de saúde, à diminuição na recuperação de ferida, ao aumento da morbidade e à fragilidade $^{1,2}$.

A diminuição do Índice de Massa Corporal (IMC) nessa população tem sido apontada como fator mais fortemente associado à mortalidade do que o excesso de peso ${ }^{3}$. Além disso, esses indivíduos apresentam risco maior de sofrer quedas e fraturas relacionadas à osteoporose ${ }^{4}$.

A prevalência de desnutrição entre idosos nas regiões brasileiras varia de $10 \%$ a $19 \%$, e esses valores são considerados marcadores de situação de pobreza em adultos pela Organização Mundial de Saúde ${ }^{5}$. Embora diversos métodos sejam empregados para avaliação do estado nutricional da população idosa, ainda é escasso o emprego de medidas da capacidade funcional como indicador do estado nutricional. Alguns estudos têm utilizado a dinamometria como um indicador funcional para avaliação da força muscular, comparado a métodos de avaliação antropométrica ${ }^{6}$.

A avaliação da capacidade funcional em idosos é importante, pois pode determinar riscos de dependência futura, quedas, morbidade e mortalidade, e são úteis para direcionar estratégias terapêuticas nessa população, tendo em vista que tais informações complementam o resultado da avaliação nutricional7,8.

No envelhecimento, observam-se mudanças na composição corporal, relacionadas à dimi- 
nuição da Massa Magra (MM) e ao aumento da gordura corporal, conhecida como Massa Gorda (MG). Alguns estudos têm procurado avaliar a influência dessas alterações sobre a diminuição da Massa Óssea (MO) $)^{9,10}$.

Apesar dos grandes prejuízos que a desnutrição ocasiona, ainda são poucos os estudos que abordam a função física e a massa óssea em idosos desnutridos. Dessa forma, o objetivo do presente estudo foi avaliar a associação do estado nutricional com a força muscular de preensão manual e a densidade mineral óssea em idosos do sexo masculino.

\section{MÉ T O D O S}

Participaram do estudo 41 idosos do sexo masculino, com idade entre 62 e 87 anos, advindos do ambulatório de geriatria do Hospital das Clínicas da Faculdade de Medicina de Ribeirão Preto (FMRPUSP) e do seu Centro de Saúde-Escola.

A amostra foi dividida em dois grupos, sendo o primeiro constituído de 20 idosos eutróficos (peso: Média - $M=69,6$, Desvio-Padrão $D P=8,4$; IMC: $M=25,7, D P=2,2)$, e o segundo, de 21 idosos desnutridos (peso: $M=50,9, D P=6,1$; IMC: $M=18,7, D P=1,8)$. A seleção dos grupos foi baseada nas informações da anamnese, exame físico e estado nutricional, classificado segundo a Mini Avaliação Nutricional (MAN) ${ }^{11}$. Foram considerados eutróficos os idosos que obtiveram pontuação igual ou superior a 23,5 pontos, e desnutridos aqueles que obtiveram pontuação igual ou inferior a 23,0 pontos.

Foram excluídos do estudo os idosos com demencia, gravemente debilitados, acamados, obesos, alcoólatras, nefropatas, hepatopatas, diabéticos, e aqueles que utilizassem próteses ortopédicas na ocasião da avaliação. Também foram excluídos os usuários de medicamentos que interferissem no metabolismo ósseo, como corticosteroide, esteroides gonadais, anticonvulsivantes e diuréticos.
O estudo foi aprovado pelo Comitê de Ética em Pesquisa do Hospital das Clínicas da Faculdade de Medicina de Ribeirão Preto da Universidade de São Paulo (Protocolo HCRP n 10.299/03). Os participantes receberam informações detalhadas a respeito dos objetivos e procedimentos do trabalho, bem como assinaram o termo de consentimento livre esclarecido.

\section{Antropometria}

O peso corporal foi medido em balança eletrônica (Filizola ID 1500, São Paulo, SP, Brasil), com capacidade de $150 \mathrm{~kg}$ e precisão de $0,1 \mathrm{~kg}$, com o indivíduo descalço e com roupas leves. A altura foi mensurada em barra metálica, graduada com precisão de $0,5 \mathrm{~cm}$, e o IMC calculado a partir de tais dados.

A Circunferência do Braço (CB) e a Circunferência da Panturrilha $(C P)$ foram mensuradas no lado direito do corpo, utilizando-se fita celuloide inextensível. A CB foi medida no ponto definido pela distância média entre a ponta do acrômio e a ponta do olecrano, e a CP no ponto definido como o de maior circunferência à inspeção.

\section{Composição corporal}

A composição corporal foi avaliada pelo método absorciometria por dupla emissão de Raios X (DXA), usando-se o modelo com análise de corpo total (Hologic, QDR 4500W, Waltham, MA, USA). A MM foi definida pela somatória do Conteúdo Mineral Ósseo (CMO,g) com as partes moles sem gordura, e a Densidade Mineral Óssea $\left(\mathrm{DMO}, \mathrm{g} / \mathrm{cm}^{2}\right)$ foi determinada no corpo inteiro. O coeficiente de variação do DXA encontrado nesse estudo foi, para o grupo eutrófico: 0,49\% para o peso corporal; $1,48 \%$ para a massa óssea; 1,99\% para a massa magra; $4,71 \%$ para a massa gorda. Já no grupo desnutrido, foi encontrado: 0,34\% para o peso corporal; $1,44 \%$ para a massa óssea; $1,79 \%$ para a massa magra; 7,98\% para a massa gorda. A ocorrência de osteoporose na 
região da coluna lombar (L1-L4), no colo do fêmur e no quadril total foi estimada de acordo com os critérios estabelecidos pela World Health Organization $(\mathrm{WHO})^{12}$.

A força muscular foi avaliada pelo teste de força de preensão manual, com a utilização do dinamômetro portátil Sammons Preston Smedley - Type Hand Dynamometer (JAMAR, Bolingbrook IL, 60440), com escala de graduação de $0-100 \mathrm{kgf}$. Durante o teste, o voluntário foi orientado a pressionar o aparelho até o alcance de sua força máxima. Foram realizadas três medições em cada membro, com intervalo mínimo de um minuto, alternando-se o lado dominante e o não-dominante, e anotando-se o maior valor.

Os dados estão descritos como média e desvio-padrão. Foi utilizado o teste - $t$ de Student para comparação das medidas antropométricas, de composição corporal, força muscular e pontuação da MAN, entre o grupo eutrófico e o desnutrido.

Para verificar a associação entre a prevalência de osteopenia e osteoporose e o estado nutricional, foi proposto o teste Exato de Fisher. Para verificar a relação entre força muscular de preensão manual e estado nutricional, foi utilizada a regressão linear simples; tal modelo de análise tem como pressuposto que seus resíduos tenham distribuição normal, com média zero e variância constante. O nível de significância adotado foi de $p<0,05^{13}$

\section{RESULTA DOS}

As características antropométricas e de composição corporal são apresentados e separada pelos grupos e nota-se, que a idade e a altura não foram diferentes entre eles. No entanto, o peso corporal, a circunferência do braço, a da panturrilha e o IMC foram significativamente menores no grupo de idosos desnutridos, da mesma forma que as variáveis $\mathrm{MM}$ e MG, avaliadas pelo método DXA, e a força muscular de preensão manual (Tabela 1).
Tabela 1. Características físicas dos idosos eutróficos e desnutridos. Ribeirão Preto (SP), 2006

\begin{tabular}{|c|c|c|c|c|}
\hline \multirow{2}{*}{ Variáveis Idosos } & \multicolumn{2}{|c|}{ Eutróficos $(n=20)$} & \multicolumn{2}{|c|}{ Desnutridos $(n=21)$} \\
\hline & M & DP & M & DP \\
\hline Idade (anos) & 73,00 & 5,40 & 76,40 & 7,00 \\
\hline Altura (m) & 1,65 & 0,50 & 1,65 & 4,00 \\
\hline Peso (Kg) & 69,60 & 8,40 & 50,90 & $6,10^{*}$ \\
\hline $\mathrm{IMC}\left(\mathrm{Kg} / \mathrm{m}^{2}\right)$ & 25,70 & 2,20 & 18,70 & $1,80^{*}$ \\
\hline $\mathrm{CB}(\mathrm{cm})$ & 29,50 & 2,60 & 23,70 & $2,40^{*}$ \\
\hline $\mathrm{CP}(\mathrm{cm})$ & 35,40 & 1,70 & 30,50 & $2,30^{*}$ \\
\hline $\mathrm{MM}(\mathrm{Kg})$ & 50,80 & 5,40 & 41,80 & $4,70^{*}$ \\
\hline$M G(K g)$ & 18,40 & 4,60 & 8,40 & $2,50^{*}$ \\
\hline $\mathrm{DMO}\left(\mathrm{g} / \mathrm{cm}^{2}\right)$ & 1,07 & 0,11 & 0,95 & $0,08^{*}$ \\
\hline FPM (Kgf) & 30,30 & 8,40 & 23,10 & $6,80^{*}$ \\
\hline MAN (0-30 pontos) & 26,80 & 1,70 & 18,00 & $3,50^{*}$ \\
\hline
\end{tabular}

*Diferença significativa entre os grupos ( $p<0,005$ teste- $t$ de Student). M: média; DP: desvio-padrão IMC: índice de massa corporal; CB: circunferência do braço; CP: circunferência da panturrilha; $M M$ : massa magra; MG: massa gorda; DMO: densidade mineral óssea; FPM: força de preensão manual; MAN: mini avaliação nutricional.

Os dados expostos na Tabela 2 mostram que entre os idosos desnutridos a presença de osteoporose foi significativamente maior $(p<0,01)$ em relação ao grupo de idosos eutróficos, em todas as regiões estudadas: colo do fêmur, $85 \%$ vs $15 \%$; quadril total, $80 \%$ vs $20 \%$; e coluna, $92 \%$ vs $8 \%$ (grupo de idosos desnutridos e eutróficos, respectivamente).

A Figura 1 ilustra a dispersão dos dados entre o grupo de idosos desnutridos e eutróficos e a força muscular de preensão manual. O modelo estimado pela regressão linear simples [FPM $=1,88+\left(1,11 \times\right.$ IMC); $\left.p<0,01 ; r^{2}=0,27\right] ;$ demonstra que, a cada diminuição do IMC, há diminuição concomitante da força muscular de preensão manual de $1,11 \mathrm{kgf}$.

\section{I S C U S S Ã O}

No presente estudo foi avaliada uma característica importante em idosos desnutridos, que é a presença de osteoporose e o prejuízo da força muscular de preensão manual. Os dados mostraram claramente que os idosos desnutridos apresentaram maior prevalência de osteoporose e menor força muscular do que os idosos eutróficos. 
Tabela 2. Frequência de osteopenia e osteoporose por região nos grupos de idosos eutróficos e desnutridos. Ribeirão Preto (SP), 2006.

\begin{tabular}{|c|c|c|c|c|c|c|c|}
\hline \multirow{2}{*}{ Região } & \multirow{2}{*}{ Classificação } & \multicolumn{2}{|c|}{ Eutróficos $(n=20)$} & \multicolumn{2}{|c|}{ Desnutridos $(n=21)$} & \multirow{2}{*}{ Total $(n=41)$} & \multirow{2}{*}{$p$-valor } \\
\hline & & $\mathrm{n}$ & $\%$ & $n$ & $\%$ & & \\
\hline \multirow[t]{4}{*}{ Colo do fêmur } & Normal & 6 & 86 & 1 & 14 & 7 & $<0,01$ \\
\hline & Osteopenia & 12 & 57 & 9 & 43 & 21 & \\
\hline & Osteoporose & 2 & 15 & 11 & 85 & 13 & \\
\hline & Total & 20 & & 21 & & 41 & \\
\hline \multirow[t]{4}{*}{ Quadril total } & Normal & 11 & 100 & 0 & 0 & 11 & $<0,01$ \\
\hline & Osteopenia & 7 & 35 & 13 & 65 & 20 & \\
\hline & Osteoporose & 2 & 20 & 8 & 80 & 10 & \\
\hline & Total & 20 & & 21 & & 41 & \\
\hline \multirow[t]{4}{*}{ Coluna } & Normal & 9 & 82 & 2 & 18 & 11 & $<0,01$ \\
\hline & Osteopenia & 10 & 56 & 8 & 44 & 18 & \\
\hline & Osteoporose & 1 & 8 & 11 & 92 & 12 & \\
\hline & Total & 20 & & 21 & & 41 & \\
\hline
\end{tabular}

$p$-valor referente ao teste Exato de Fisher.

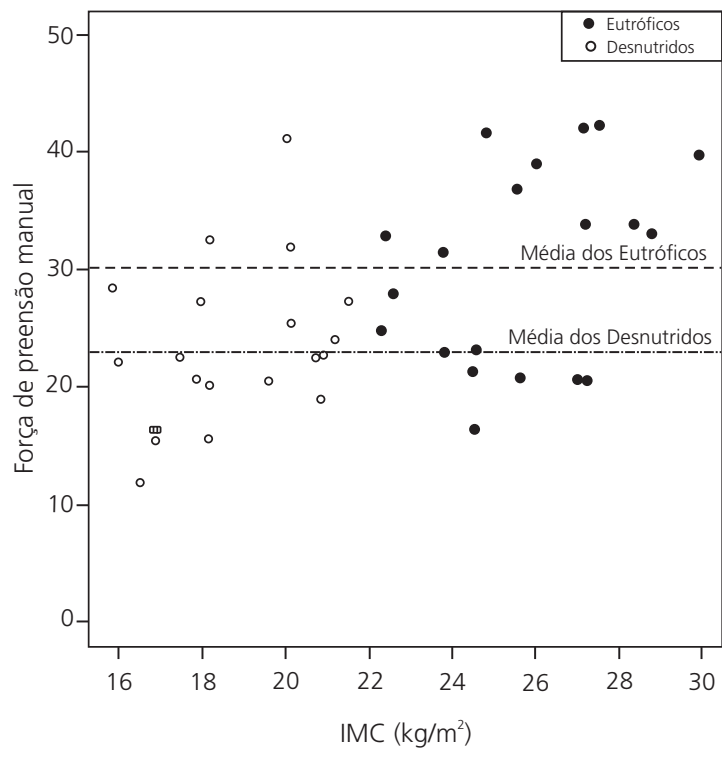

Figura 1. Dispersão entre IMC e força muscular de preensão manual por grupo. Ribeirão Preto (SP), 2006.

De acordo com alguns autores, a desnutrição pode ser considerada uma síndrome geriátrica, devido a sua relação com transtornos mentais (depressão e comprometimento cognitivo), doenças somáticas, além de ser uma das maiores causas de declínio funcional e aumento da morbidade e mortalidade nessa população ${ }^{14}$. Baseado nessas informações, os achados do presente trabalho demonstram a importância de se avaliarem aspectos multidimensionais no idoso, especialmente os desnutridos, complementando os dados comumente avaliados sobre o estado nutricional, como os exames bioquímicos, antropométricos e de composição corporal ${ }^{15}$.

O grupo de idosos desnutridos apresentou menor densidade mineral óssea em comparação com os idosos eutróficos $(p<0,01)$. Além disso, houve associação significativa entre a prevalência de osteopenia e osteoporose e o estado nutricional comprometido, sendo que grande parte dos idosos desnutridos apresentaram T-score abaixo de -2,5 desvio-padrão. Esses dados são consis-tentes com o diagnóstico de osteoporose segundo os critérios adotados pela World Health Organization ${ }^{12}$ e enfatizam o alto risco de fraturas nessa população. Estudos anteriores observaram também associação entre peso corporal e DMO, tanto na coluna como no colo do fêmur ${ }^{16,17}$. Além disso, outro importante estudo também identificou associação entre idosos com baixo peso corporal e osteoporose nas regiões do colo do fêmur e quadril total, com T-score de -3 e -2,7, respectivamente ${ }^{18}$, demonstrando a influência do peso corporal na massa óssea.

Existem evidências de que há prejuízos na função muscular e diminuição da força, na presença da desnutrição ${ }^{19}$. Newman et al. ${ }^{20}$ demons- 
traram, de modo similar ao presente estudo, que a desnutrição está associada à diminuição da massa magra e que tal variável está intimamente relacionada à força, podendo interferir na função do idoso.

De acordo com alguns estudos, o prejuízo na força muscular dos pacientes desnutridos surge antes das alterações laboratoriais e antropométricas ${ }^{21,22}$. Por essa razão, a avaliação funcional do idoso desnutrido é de suma importância para acompanhar seu estado nutricional e as intervenções terapêuticas ${ }^{19}$.

Neste trabalho, os valores da força muscular de preensão manual dos idosos eutróficos foram condizentes com os valores de referência encontrados em estudo brasileiro ${ }^{23}$, ao passo que a força muscular dos idosos desnutridos foi significativamente menor. Esses achados concordam com estudos que avaliaram a capacidade funcional e a força muscular de pacientes desnutridos e observaram também pior desempenho associado à desnutrição ${ }^{24,25}$.

A dinamometria é um método seguro, rápido, prático, barato e não invasivo de avaliação de força isométrica dos membros superiores. Segundo Frederiksen et al. ${ }^{26}$, é aplicado também como preditor de incapacidade, morbidade e mortalidade nos idosos. A força de preensão manual é como um teste funcional indicador de depleção proteica ${ }^{27} \mathrm{e}$, nesse sentido, tem sido utilizada como indicador funcional de desnutrição nesses indivíduos.

Os dados da análise de regressão linear apontaram que, a cada diminuição do IMC, há diminuição da força muscular de preensão manual, e esses dados auxiliam na confirmação da relação entre o peso corporal e a força muscular desenvolvida pelo indivíduo. Com o avançar da idade e com o menor peso corporal, aumentam as chances de desenvolvimento da sarcopenia, processo que tem impacto direto na força e na funcionalidade do idoso ${ }^{28}$. Estudos anteriores encontraram correlação positiva entre a força muscular e o IMC em idosos ${ }^{27}$.
A literatura aponta o comprometimento do estado nutricional e a perda de peso como responsáveis pela alteração na função do músculo esquelético e, consequentemente, pela perda de força muscular. Por isso, recentemente, alguns estudos têm comparado parâmetros antropométricos e funcionais, reforçando a validade desses indicadores como instrumentos de avaliação nutricional29,30.

Diante dos resultados encontrados nesta pesquisa e da grande relevância clínica do tema, é importante que futuros estudos sejam conduzidos com maior número de voluntários, utilizando diferentes métodos de avaliação nutricional e outras maneiras de avaliar a capacidade funcional nessa população.

Sugere-se que intervenções públicas de saúde sejam implementadas para acompanhar precocemente a evolução do estado nutricional e funcional de idosos, em especial a perda de força muscular e do compartimento ósseo, a fim de prevenir complicações advindas da desnutrição nessa população.

\section{O N C L U S Ã O}

Os resultados do presente estudo indicam que a desnutrição está associada à menor força muscular de preensão manual e à osteoporose no idoso.

Nesse sentido, é importante que seja incluída a avaliação da força muscular de preensão manual nas avaliações nutricionais, por ser um indicador funcional que complementa a avaliação do estado nutricional de idosos.

\section{A GRADECIMENTO}

Pelo apoio financeiro da Coordenação de Aperfeiçoamento de Pessoal de Nível Superior (CAPES).

\author{
C O LABORADORES \\ K.H.C. VILAÇA, E. FERRIOLLI, J.S. MARCHINI e \\ J.C. MORIGUTI contribuíram na concepção do projeto,
}


análise dos dados e revisão do artigo. N.K.C. LIMA e F.J.A. PAULA contribuíram na análise dos dados e revisão do artigo.

\section{REFERÊ NCIAS}

1. Moriguti JC, Moriguti EKU, Ferriolli E, Cação JC, lucif Junior N, Marchini JS. Involuntary weight loss in elderly individuals: assessment and treatment. São Paulo Med J. 2001; 119(2):72-7. doi: 10.1590/ S1516-31802001000200007.

2. Banks M, Bauer J, Graves N, Ash S. Malnutrition and pressure ulcer risk in adults in Australian health care facilities. Nutrition. 2010; 26(9):896-901. doi: 10.1016/j.nut.2009.09.024.

3. Sergi G, Perissinotto E, Pisent C, Buja A, Maggi S, Coin A, et al. An adequate threshold for body mass index to detect underweight condition in elderly persons: the Italian longitudinal study on aging. J Gerontol A Biol Sci Med Sci. 2005; 60(7):866-71. doi: 10.1093/gerona/60.7.866.

4. Ozeraitiene $\mathrm{V}$, Butenaite $\mathrm{V}$. The evaluation of bone mineral density based on nutritional status, age, and anthropometric parameters in elderly women. Medicina (Kaunas). 2006; 42(10):836-42.

5. Otero UB, Rozenfeld S, Gadelha AMJ, Carvalho MS. Mortalidade por desnutrição em idosos, região Sudeste do Brasil, 1980-1997. Rev Saúde Pública. 2002; 36(2):141-8. doi: 10.1590/S0034-89102002 000200004.

6. Luna-Heredia E, Martín-Peña G, Ruiz-Galiana J. Handgrip dynamometry in healthy adults. Clin Nutr. 2005; 24(2):250-8. doi: 10.1016/j.clnu.2004.10.0 07.

7. Gaskill D, Black L J, Isenring EA, Hassal S, Sanders $\mathrm{F}$, Bauer J. Malnutrition prevalence and nutrition issues in residential aged care facilities. Aust J Ageing. 2009; 27(4):189-94. doi: 10.1111/j.1741-6 612.2008.00324.x.

8. Jakobsen LH, Rask IK, Kondrup J. Validation of handgrip strength and endurance as a measure of physical function and quality of life in healthy subjects and patients. Nutrition. 2010; 26(5): 542-50. doi: 10.1016/j.nut.2009.06.015.

9. Baumgartner RN, Stauber PM, Koehler KM, Romero L, Garry PJ. Associations of fat and muscle masses with bone mineral in elderly men and women. Am J Clin Nutr. 1996; 63(3):365-72.

10. Pereira FA, Castro JAS, Santos JE, Foss MC, Paula FJA. Impact of marked weight loss induced by bariatric surgery on bone mineral density and remodeling. Braz J Med Biol Res. 2007; 40(4): 509-17. doi: 10.1590/S0100-879X2006005000 073.
11. Guigoz Y, Vellas B, Garry PJ. Mini nutritional assessment: a practical assessment tool for grading the nutritional state of elderly patients. Facts Res Gerontol. 1994; (Suppl 2):15-59.

12. World Health Organization. Expert Committee. Assessment of fracture risk and its application to screening for postmenopausal osteoporosis. Geneva: WHO; 1994. WHO Technical Report Series; 843.

13. Montgomery DC. Design and analysis of experiments. New York: John Wiley-Sons; 1996.

14. Rikkert MGMO, Rigaud AS. Malnutrition research: high time to change the menu. Age Ageing. 2003; 32(3):241-3. doi: 10.1093/ageing/32.3.241.

15. Sampaio LR. Avaliação nutricional e envelhecimento. Rev Nutr. 2004; 17(4):507-14. doi: 10.15 90/S1415-52732004000400010.

16. Lewin S, Gouvea CHA, Marone MMS, Wehba S, Malvestiti LF, Bianco AC. Densidade mineral óssea vertebral e femoral de 724 mulheres brancas brasileiras: influência da idade e do peso corporal.Rev Assoc Med Bras. 1997; 43(2):127-36.doi: 10.1590/S0104-42301997000200009.

17. Edelstein SL, Barrett-Connor E. Relation between body size and bone mineral density in elderly men and women. Am J Epidemiol. 1993; 138(3):160-9.

18. Coin A, Sergi G, Beninca P, Lupoli L, Cinti G, Ferrara $L$, et al. Bone mineral density and body composition in underweight and normal elderly subjects. Osteoporosis Int. 2000; 11(12):1043-50. doi: 10.1 007/s001980070026.

19. Norman K, Stobäus N, Gonzalez MC, Schulzke JD, Pirlich M. Hand grip strength: outcome predictor and marker of nutritional status. Clin Nutr. 2011; 30(2):135-42. doi: 10.1016/j.clnu.2010.09.010.

20. Newman AB, Haggerty $C L$, Goodpaster B, Harris T, Kritchevsky S, Nevitt $M$, et al. Strength and muscle quality ins a well-functioning cohort of older adults: the health, aging and body composition study. J Am Geriatr Soc. 2003; 51(3):323-30. doi: 10.1046/ j.1532-5415.2003.51105.x.

21. Russell DM, Leiter LA, Whitwell J, Marliss EB, Jeejeebhoy KN. Skeletal muscle function during hypocaloric diets and fasting: a comparison with standard nutritional assessment parameters. Am J Clin Nutr. 1983; 37(1):133-8.

22. Lopes J, Russell DM, Whitwell J, Jeejeebhoy KN. Skeletal muscle function in malnutrition. Am J Clin Nutr. 1982; 36(4):602-10.

23. Budziareck MB, Duarte RRP, Barbosa-Silva MC. Reference values and determinants for handgrip strength in healthy subjects. Clin Nutr. 2008; 27(3): 357-62. doi: 10.1016/j.clnu.2008.03.008. 
852 | K.H.C. VILAÇA et al

24. Álvares-da-Silva MR, Silveira TR. Comparison between handgrip strength, subjective global assessment, and prognostic index in assessing malnutrition and predicting clinical outcome in cirrhotic outpatients. Nutrition. 2005; 21(2):113-7. doi: 10.1016/j.nut.2004.02.002.

25. Kruizenga HM, Jonge $P$, Seidell JC, Neelemaat $F$, Bodegraven AA, Wierdsma NJ, et al. Are malnourished patients complex patients? health status and care complexity of malnourished patients detected by the Short Nutritional Assessment Questionnaire. Eur J Intern Med. 2006; 17(3):189-94. doi:10.1016/j.ejim.2005.11.019.

26. Frederiksen H, Hjelmborg J, Mortensen J, McGue M, Vaupel JW, Christensen K. Age trajectories of grip strength: cross-sectional and longitudinal data among 8,342 Danes aged 46-102. Ann Epidemiol. 2006; 16(7):554-62. doi: 10.1016/j.annepidem. 2005.10.006.

27. Figueiredo FA, Dickson ER, Pasha TM, Porayko MK, Therneau TM, Malinchoc M, et al. Utility of standard nutritional parameters in detecting body cell mass depletion in patients with end-stage liver disease. Liver Transpl. 2000; 6(5):575-81. doi: 10.1 053/jlts.2000.9736.

28. Cruz-Jentoft AJ, Baeyens JP, Bauer JM, Boirie Y, Cederholm T, Landi F, et al. Sarcopenia: European consensus on definition and diagnosis: report of the European working group on sarcopenia in older people. Age Ageing. 2010; 39(4):412-23. doi: 10.1 093/ageing/afq034.

29. Jeejeebhoy KN. Nutritional assessment. Nutrition. 2000; 16(7-8):585-90.

30. Schlussel MM, Anjos LA, Kac GC. A dinamometria manual e seu uso na avaliação nutricional. Rev Nutr. 2008; 21(2):233-5. doi: 10.1590/S1415-527320 080002200009 .

Recebido em: 20/5/2010

Versão final reapresentada em: 31/8/2011 Aprovado em: 20/9/2011 